ISSN 0103-9954

\title{
TÉCNICAS MULTIVARIADAS APLICADAS À AVALIAÇÃO DE RESÍDUOS LIGNOCELULÓSICOS PARA A PRODUÇÃO DE BIOENERGIA
}

\section{MULTIVARIATE TECHNIQUES APPLIED TO EVALUATION OF LIGNOCELLULOSIC RESIDUES FOR BIOENERGY PRODUCTION}

\author{
Thiago de Paula Protásio ${ }^{1}$ Lina Bufalino ${ }^{2}$ Mario Guimarães Junior ${ }^{3}$ Gustavo Henrique Denzin Tonoli ${ }^{4}$ \\ Paulo Fernando Trugilho ${ }^{4}$
}

\section{RESUMO}

Na avaliação de resíduos lignocelulósicos para a produção de bioenergia é necessário considerar as várias características e propriedades que podem ser correlacionadas. Este fato demanda o emprego de diversas técnicas de análise multivariada que possibilitem a avaliação dos fatores energéticos mais relevantes. O objetivo deste trabalho foi aplicar as análises de agrupamento e componentes principais na seleção e avaliação de resíduos lignocelulósicos visando à produção de bioenergia. Foram utilizados 8 tipos de biomassa residual, dos quais foram determinados os teores dos componentes elementares $(\mathrm{C}, \mathrm{H}, \mathrm{O}, \mathrm{N}, \mathrm{S})$, os teores de lignina, extrativos totais e cinzas, a densidade básica e o poder calorífico superior e inferior. As duas técnicas multivariadas empregadas para a avaliação e seleção de resíduos lignocelulósicos para a produção de energia foram eficientes, sendo observadas similaridades entre os grupos de biomassa formados por elas. Pela interpretação da primeira componente principal obtida, criou-se um índice de desempenho global para avaliar a viabilidade do aproveitamento energético da biomassa. A interpretação da segunda componente principal permitiu um contraste entre os teores de nitrogênio e enxofre e o teor de oxigênio.

Palavras-chave: análise multivariada; biomassa; energia.

\begin{abstract}
The evaluation of lignocellulosic wastes for bioenergy production demands to consider several characteristics and properties that may be correlated. This fact demands the use of various multivariate analysis techniques that allow the evaluation of relevant energetic factors. This work aimed to apply cluster analysis and principal components analyses for the selection and evaluation of lignocellulosic wastes for bioenergy production. 8 types of residual biomass were used, whose the elemental components $(\mathrm{C}, \mathrm{H}, \mathrm{O}, \mathrm{N}, \mathrm{S})$ content, lignin, total extractives and ashes contents, basic density and higher and lower heating values were determined. Both multivariate techniques applied for evaluation and selection of lignocellulosic wastes were efficient and similarities were observed between the biomass groups formed by them. Through the interpretation of the first principal component obtained, it was possible to create a global development index for the evaluation of the viability of energetic uses of biomass. The interpretation of the second principal component allowed a contrast between nitrogen and sulfur contents with oxygen content.
\end{abstract}

Keywords: multivariate analysis; biomass; energy.

1 Engenheiro Florestal, Mestrando em Ciência e Tecnologia da Madeira, Departamento de Ciências Florestais, Universidade Federal de Lavras, Câmpus Universitário, Caixa Postal 3037, CEP 37200-000, Lavras (MG), Brasil. depaulaprotasio@gmail.com

2 Engenheira Florestal, Doutoranda em Ciência e Tecnologia da Madeira, Departamento de Ciências Florestais, Universidade Federal de Lavras, Câmpus Universitário, Caixa Postal 3037, CEP 37200-000, Lavras (MG), Brasil. linabufalino@yahoo.com.br

3 Licenciado Ciências e Matemática, Msc., Professor do Departamento de Eletrônica, CEFET-MG, Câmpus Araxá, Av. Ministro Olavo Drummond, 25, São Geraldo, CEP38180-510, Araxá(MG), Brasil.mgjunior@araxa.cefetmg.br

4 Engenheiro Florestal, Dr., Professor do Departamento de Ciências Florestais, Universidade Federal de Lavras, Câmpus Universitário, Caixa Postal 3037, CEP 37200-000, Lavras (MG), Brasil. gustavotonoli@ufla.br / trugilho@ufla.br

Recebido para publicação em 29/03/2011 e aceito em 22/08/2012 


\section{INTRODUÇÃO}

A evolução do consumo mundial de energia baseada em combustíveis não renováveis conduziu a humanidade a uma matriz energética insegura e poluidora. O Brasil, por outro lado, tem aproveitado seu potencial de produção agrícola e florestal para a utilização de energia renovável. O país apresenta atualmente quase $50 \%$ de sua matriz energética proveniente de fontes renováveis, sendo $15,2 \%$ correspondente à hidroeletricidade e $32 \%$ a diversos tipos de biomassa (MME, 2010). Nesse contexto, a utilização de resíduos lignocelulósicos para a produção de energia pode ser uma boa fonte de energia renovável, dada a grande quantidade de biomassa residual que é produzida pelo setor agroflorestal.

Na seleção e classificação de resíduos lignocelulósicos para a produção de bioenergia torna-se necessária a avaliação de características e propriedades que geralmente apresentam alguma relação entre si e com o poder calorífico, variável energética de alta relevância para o aproveitamento energético da biomassa residual, pois expressa a quantidade de energia térmica que é liberada durante a combustão completa de uma unidade de massa ou de volume do combustível (PROTÁSIO et al., 2011; BRAND, 2010; EROL et al., 2010; AKKAYA, 2009; HUANG et al., 2009; MAJUMDER et al., 2008; SHENG e AZEVEDO, 2005; FRIEDL et al., 2005; PARIKH et al., 2005; DEMIRBAS e DEMIRBAS, 2004; CORDERO et al., 2001; DEMIRBAS, 2001). Desse modo, na seleção de resíduos lignocelulósicos para a geração de bioenergia, pode ser fundamental empregar técnicas estatísticas multivariadas para reconhecer as propriedades químicas e físicas mais importantes para um bom rendimento energético e entender as semelhanças entre as diferentes biomassas avaliadas.

Nesse contexto, a análise de componentes principais (ACP) objetiva explicar ou modelar a estrutura de variância e covariância de um vetor aleatório composto de variáveis aleatórias, pela construção de combinações lineares das variáveis originais. A ACP deve ser entendida como uma técnica de análise intermediária pela qual é possível obter um número de componentes principais igual ao número de variáveis originais, mas geralmente se deseja obter uma redução do número de variáveis analisadas e uma interpretação das combinações lineares construídas. Uma grande vantagem dessa técnica multivariada é a não exigência de normalidade do vetor aleatório em estudo (FERREIRA, 2008; MINGOTI, 2005; KENT e COKER, 1992).
As componentes principais obtidas são na realidade variáveis latentes e não são correlacionadas, diferentemente das variáveis originais observadas (FERREIRA, 2008; MINGOTI, 2005). Segundo Hair Jr. et al. (2009), um pouco de multicolinearidade é desejável nessa análise estatística, ou seja, essa técnica multivariada será tão eficaz quanto maior for a correlação entre as variáveis originais. A primeira variável latente obtida apresenta o maior autovalor e, consequentemente, a maior variância. A segunda variável latente apresenta o segundo maior autovalor e assim sucessivamente, sendo necessário um critério para reter apenas algumas dessas variáveis latentes, de tal forma que tenhamos uma grande porção da variância total explicada por um pequeno conjunto de novas variáveis (FERREIRA, 2008; MINGOTI, 2005).

Já a análise de agrupamento ou cluster, tem como objetivo agregar os objetos com base nas suas características. Os objetos da amostra são divididos em grupos de forma que os elementos pertencentes a um mesmo grupo sejam similares entre si em relação às variáveis ou características que neles foram avaliadas e os elementos de grupos diferentes sejam heterogêneos (ou dissimilares) em relação a essas mesmas características (HAIR JR. et al., 2009; FERREIRA, 2008; MINGOTI, 2005; JOHNSON e WICHERN, 1992; MARRIOTT, 1974).

Apesar de Protásio et al. (2011), Caixeta et al. (2003), Friedl et al. (2005) e Gómez et al. (2007) mostrarem o potencial de algumas técnicas multivariadas na avaliação das características relacionadas à biomassa vegetal, ainda existe ausência na literatura de trabalhos envolvendo as análises de agrupamento e de componentes principais na avaliação e seleção de resíduos lignocelulósicos para a produção de bioenergia.

Portanto, o objetivo do trabalho foi aplicar as técnicas de análises multivariadas de agrupamento e componentes principais na seleção e avaliação de resíduos lignocelulósicos visando à produção de bioenergia.

\section{MATERIAL E MÉTODOS}

\section{Caracterização dos resíduos lignocelulósicos}

Foram utilizados resíduos lignocelulósicos oriundos da produção florestal e agrícola compostos por maravalhas de eucalipto (Eucalyptus urophylla de 6 anos de idade), maravalhas de cedro australiano (Toona ciliata de 18 anos de idade), maravalhas 
de pinus (Pinus spp. de 35 anos de idade), casca de arroz (Oryza sativa), bagaço de cana-de-açúcar (Saccharum officinarum), casca e pergaminho de café (Coffea arabica) (resíduos do processamento dos grãos), resíduos da colheita do milho (Zea mays) (palha, sabugo, caule e folhas) e polpa celulósica residual de bambu (Bambusa vulgaris).

Por meio da análise elementar realizada em um analisador universal da marca Elementar (modelo Vario Micro Cube), foi possível a quantificação dos teores de carbono, hidrogênio, nitrogênio e enxofre em relação à massa seca dos resíduos lignocelulósicos. As amostras foram moídas e peneiradas, sendo utilizada para o ensaio a fração que passou pela peneira de 60 mesh e ficou retida na peneira de 270 mesh. Amostras de $2 \mathrm{mg}$ de cada resíduo foram completamente incineradas a $1.200^{\circ} \mathrm{C}$. O analisador utiliza como gases de arraste e ignição o hélio e o oxigênio, respectivamente. $\mathrm{O}$ teor de oxigênio foi obtido por diferença, segundo a Eq. 1 (BECH et al., 2009):

$$
\mathrm{O}=100-\mathrm{C}-\mathrm{H}-\mathrm{N}-\mathrm{S}-\mathrm{A}
$$

Onde: O é o teor de oxigênio (\%); C é o teor de carbono (\%); H é o teor de hidrogênio (\%); N é o teor de nitrogênio (\%); S é o teor de enxofre (\%); A é o teor de cinzas (\%).

$\mathrm{O}$ teor de cinzas foi determinado segundo as diretrizes previstas na norma ABTCP M11/77 (1974a) com o uso de um forno tipo mufla. Seguindo as determinações das normas ABTCP M3/69 (1974b) e ABTCP M70/71 (1974c), foram quantificados os extrativos totais e a lignina presente nos resíduos lignocelulósicos estudados, respectivamente.

Para a quantificação do poder calorífico superior (PCS) foi utilizado um calorímetro digital da marca IKA C-200. A determinação do poder calorífico superior foi realizada seguindo os procedimentos recomendados pela NBR 8633 (ABNT, 1984). O poder calorífico inferior (PCI) foi estimado utilizando a Eq. 2:

$$
\mathrm{PCI}=\mathrm{PCS}-5,72 \times(9 \times \mathrm{H}+\mathrm{U})
$$

Onde: PCI é o poder calorífico inferior (kcal/kg); PCS é o poder calorífico superior $(\mathrm{kcal} /$ $\mathrm{kg}$ ); $\mathrm{H}$ é o teor de hidrogênio (\%); U é a umidade em base seca (\%).

A densidade básica foi determinada pelo método da proveta ou método do deslocamento de água, preconizado por Azzini et al. (1981) e base- ado nos preceitos do Princípio de Arquimedes. As amostras, com aproximadamente $10 \mathrm{~g}$, previamente saturadas, foram imersas em uma proveta graduada com capacidade de $250 \mathrm{~mL}$, contendo $200 \mathrm{~mL}$ de água. Após a imersão, registrou-se o volume de água translocada, que corresponde ao volume da amostra, considerando a densidade da água igual a $1 \mathrm{~g} / \mathrm{cm}^{3}$. Posteriormente, levaram-se as amostras à estufa de circulação forçada a $103 \pm 2{ }^{\circ} \mathrm{C}$, para a obtenção da massa seca. Todas as análises foram realizadas considerando 5 repetições amostradas aleatoriamente totalizando 40 observações por variável.

\section{Estatísticas descritivas aplicadas às variáveis originais}

A média, mediana, desvio padrão, coeficiente de variação e os valores de máximo e mínimo encontrados para as características dos resíduos lignocelulósicos foram determinados com o intuito de avaliar a variabilidade da biomassa analisada e o comportamento das variáveis originais.

\section{Análise de agrupamento}

Utilizou-se a distância euclidiana como medida de proximidade e o método da ligação média (UPGMA) como técnica hierárquica aglomerativa, ou seja, a distância entre grupos foi calculada pela distância média entre todos os pares de objetos dos dois diferentes grupos (FERREIRA, 2008).

A qualidade do dendrograma obtido foi avaliada através do coeficiente de correlação cofenética que mede a correlação entre as distâncias recuperadas do dendrograma com a matriz de distâncias originais entre os resíduos lignocelulósicos. Foram consideradas as médias aritméticas de todas as variáveis originais mensuradas nos resíduos lignocelulósicos nessa análise multivariada.

\section{Análise de componentes principais}

Foram utilizadas apenas as médias de cada variável e a análise de componentes principais foi realizada considerando-se a matriz de correlação dos dados. Ferreira (2008) e Mingoti (2005) afirmam que as componentes principais obtidas via matriz de covariâncias são influenciadas pelas variáveis de maior variância ou pelo efeito da escala das variáveis, sendo que esse problema pode ser amenizado se as componentes forem obtidas por meio da matriz de correlação dos dados originais. Esse pro- 
cedimento equivale a padronizar as variáveis e permite maior acurácia na análise (MINGOTI, 2005).

O número de componentes principais consideradas e interpretadas foi determinado pelo gráfico denominado scree plot, sendo que na abscissa encontram-se as componentes principais e na ordenada os autovalores que representam a variância estimada para cada componente principal. A ideia é analisar esse tipo de gráfico e avaliar onde o acréscimo de um componente principal não provoca alterações expressivas na variância, ou seja, o segmento de reta atingiu um patamar onde o coeficiente angular se aproxima de zero (FERREIRA, 2008).

Por meio da interpretação das componentes principais e criação de índices de interesse (MINGOTI, 2005) estimaram-se os escores das duas primeiras componentes como forma de classificar os resíduos para a produção de bioenergia.

Segundo Hair Jr. et al. (2009), um pouco de multicolinearidade é desejável nessa análise estatística justificando assim o emprego das variáveis originais mais correlacionadas, ou seja, os teores de cinzas, carbono, hidrogênio, enxofre, oxigênio, poder calorífico superior (PCS) e poder calorífico inferior (PCI). Todas as análises estatísticas foram efetuadas utilizando-se o software estatístico R versão 2.11.0, pacote stats (R DEVELOPMENT CORE TEAM, 2010).

\section{RESULTADOS E DISCUSSÃO}

\section{Estatísticas descritivas aplicadas às variáveis originais}

Na Figura 1 encontram-se as estatísticas descritivas aplicadas aos teores de oxigênio, carbono e hidrogênio dos resíduos lignocelulósicos avaliados.

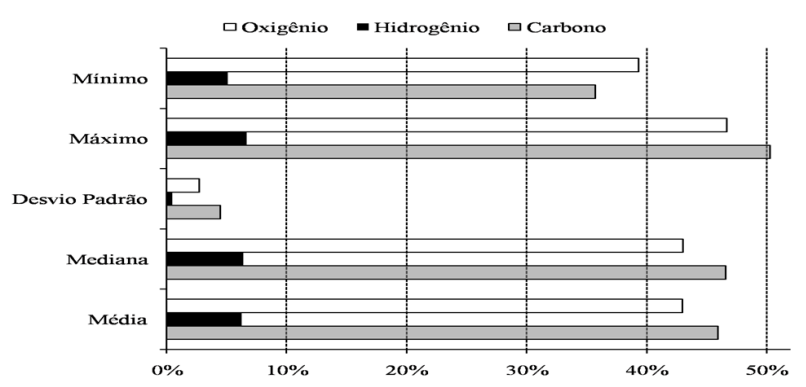

FIGURA 1: Estatísticas descritivas aplicadas ao C, $\mathrm{H}$ e $\mathrm{O}$ dos resíduos lignocelulósicos.

FIGURE 1: Descriptive statistics applied to C, $\mathrm{H}$ and $\mathrm{O}$ of lignocellulosic residues.
Os valores médios dos teores de $\mathrm{C}, \mathrm{H}$ e $\mathrm{O}$ dos resíduos lignocelulósicos avaliados foram de 45,93, 6,24 e 42,97\%, respectivamente. Esse resultado demonstra que os principais elementos constituintes da biomassa vegetal são o $\mathrm{C}$ e o $\mathrm{O}$, sendo encontrados baixos valores de H. Além disso, esses constituintes elementares juntamente com os teores de lignina, extrativos totais e cinzas são fundamentais para a seleção de materiais lignocelulósicos para a produção de energia, pois influenciam diretamente o poder calorífico dos materiais, seja positiva ou negativamente (PAULA et al., 2011; PROTÁSIO et al., 2011; EROL et al., 2010; AKKAYA, 2009; HUANG et al., 2009; MAJUMDER et al., 2008; SHENG e AZEVEDO, 2005; FRIEDL et al., 2005; PARIKH et al., 2005; DEMIRBAS e DEMIRBAS, 2004; CORDERO et al., 2001; DEMIRBAS, 2001; NORDIN, 1994; KUMAR et al., 1992).

O menor $(39,32 \%)$ e o maior $(46,67 \%)$ teores de $\mathrm{O}$ foram encontrados para os resíduos da colheita do milho e para a polpa celulósica de bambu, respectivamente, sendo essa uma característica importante para a seleção de resíduos lignocelulósicos para a produção de bioenergia, uma vez que altos teores de oxigênio decrescem o poder calorífico dos materiais (PAULA et al., 2011; PROTÁSIO et al., 2011; HUANG et al., 2009; NORDIN, 1994). Quanto ao teor de H, o menor $(5,11 \%)$ e o maior $(6,65 \%)$ teores foram encontrados para a casca de arroz e as maravalhas de pinus, respectivamente, sendo essa característica primordial para a avaliação de materiais para a geração de energia, pois o $\mathrm{H}$ é um elemento altamente energético e apresenta correlação positiva e direta com o poder calorífico (PROTÁSIO et al., 2011; DEMIRBAS e DEMIRBAS, 2004).

A casca de arroz apresentou o menor teor de $\mathrm{C}(35,74 \%)$ e as maravalhas de cedro australiano o maior teor $(50,28 \%)$. O carbono é um dos principais constituintes da biomassa e essencial para a produção energética. Nota-se que a casca de arroz apresentou as piores características para a produção de bioenergia e os resíduos de origem florestal (cedro e pinus) apresentaram características relevantes para a sua utilização energética. Os resíduos lignocelulósicos avaliados apresentaram homogeneidade (baixo desvio padrão) quanto aos teores $\mathrm{C}, \mathrm{H}$ e $\mathrm{O}$ e baixos coeficientes de variação (PIMENTELGOMES, 2009), inferiores a $10 \%$.

$\mathrm{Na}$ Figura 2 encontram-se as estatísticas descritivas aplicadas aos teores de enxofre e nitrogênio da biomassa residual avaliada. 


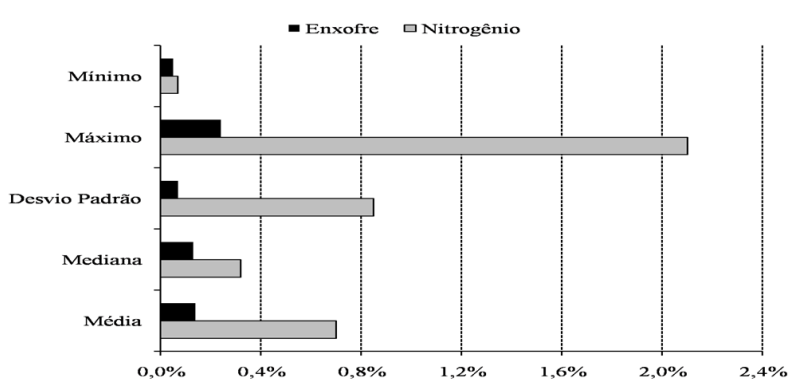

FIGURA 2: Estatísticas descritivas aplicadas ao $\mathrm{S} \mathrm{e}$ $\mathrm{N}$ dos resíduos lignocelulósicos.

FIGURE 2: Descriptive statistics applied to $\mathrm{S}$ and $\mathrm{N}$ of lignocellulosic residues.

Altos teores de $\mathrm{N}$ e $\mathrm{S}$ são indesejáveis, pois não contribuem para a maximização do poder calorífico e resultam em danos ambientais como a formação de óxidos de nitrogênio tóxicos (no caso da liberação de N), formação de chuva ácida e corrosão (KUMAR et al., 2010; BILGEN e KAYGUSUZ, 2008).

O valor médio encontrado para o teor de $\mathrm{S}$ foi baixo $(0,15 \%)$, sendo o contrário observado para o teor de N (0,70\%). Segundo Brand (2010), o teor médio de $\mathrm{N}$ para folhosas é de $0,30 \%$, ou seja, inferior ao encontrado para os resíduos lignocelulósicos avaliados. Nota-se que o desvio padrão para o teor de nitrogênio da biomassa avaliada foi alto, bem como o coeficiente de variação $(121,4 \%)$ o que indica alta variabilidade dos resíduos lignocelulósicos quanto a essa característica. Observou-se que o menor $(0,07 \%)$ e o maior $(2,10 \%)$ teores de $\mathrm{N}$ foram encontrados para as maravalhas de eucalipto e para a casca de café, respectivamente, o que justifica o elevado coeficiente de variação encontrado.

Para o teor de $\mathrm{S}$ observou-se que os valores mínimo $(0,05 \%)$ e máximo $(0,24 \%)$ foram determinados para a polpa celulósica residual de bambu e para os resíduos do processamento dos grãos de café, respectivamente. O coeficiente de variação encontrado para o teor de $\mathrm{S}$ foi de $50 \%$, o que reflete a variabilidade da biomassa analisada quanto a essa característica. Esses resultados demonstram que a utilização energética dos resíduos do processamento dos grãos de café pode ser realizada tomando-se medidas que previnam possíveis danos ambientais.

As estatísticas descritivas aplicadas à análise química molecular dos resíduos lignocelulósicos avaliados encontram-se na Figura 3.

Considerando-se a análise química molecular dos resíduos lignocelulósicos, o teor de lignina apresentou a maior variação correspondendo a um alto desvio padrão $(7,06 \%)$ e a um alto coeficiente de variação $(28,49 \%)$. Os teores de cinzas e extrativos totais também apresentaram altos coeficientes de variação (PIMENTEL-GOMES, 2009): 138,48 e $73,71 \%$, respectivamente, sendo que essas características são relevantes na avaliação energética dos materiais combustíveis. Os elevados coeficientes de variação encontrados são consequência da heterogeneidade da biomassa, uma vez que foram avaliados resíduos provenientes das atividades agrícolas e florestais e que pertencem a famílias botânicas distintas.

De maneira geral, os resíduos lignocelulósicos apresentaram alto teor médio de cinzas (4,08\%), sendo este resultado proporcionado pela constituição inorgânica dos resíduos agrícolas. O teor de extrativos totais e lignina também apresentaram valores elevados, em média, 8,56 e $24,78 \%$, respectivamente. Elevados teores de cinzas ou de minerais decrescem o poder calorífico, enquanto que altos teores de extrativos totais e lignina tendem a aumentá-lo (TELMO e LOUSADA, 2011; BRAND, 2010; DEMIRBAS, 2001).

Shafizadeh (1981) afirma que os extrativos totais e a lignina apresentam alto calor de combustão e baixo grau de oxidação quando comparados com a holocelulose, o que justificaria a influência positiva desses componentes químicos no poder calorífico. Além disso, a lignina é um polímero que apresenta alto teor de carbono e é resistente à decomposição térmica quando comparado à celulose e às hemiceluloses (GANI e NARUSE, 2007).

$\mathrm{O}$ menor teor de cinzas foi encontrado para as maravalhas de eucalipto $(0,25 \%)$, enquanto que a casca de arroz apresentou o maior valor $(16,78 \%)$.

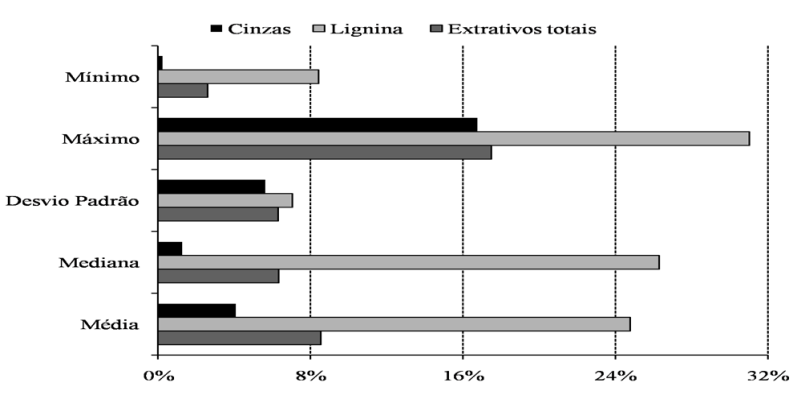

FIGURA 3: Estatísticas descritivas aplicadas à análise química dos resíduos lignocelulósicos.

FIGURE 3: Descriptive statistics applied to chemical analysis of lignocellulosic residues. 
Quanto ao teor de extrativos totais, as maravalhas de pinus e os resíduos da colheita do milho apresentaram o menor $(2,60 \%)$ e o maior $(17,50 \%)$ valores, respectivamente. A polpa celulósica residual de bambu apresentou o menor teor de lignina $(8,42 \%)$ e os resíduos do processamento do café o maior teor $(31,03 \%)$

$\mathrm{Na}$ Figura 4 encontram-se as estatísticas descritivas aplicadas às variáveis energéticas (PCS e PCI) e a densidade básica (DB) da biomassa residual avaliada.

A densidade básica é uma característica muito útil para comparações entre diferentes biomassas. Observa-se que os resíduos lignocelulósicos apresentaram densidade básica média de $175,08 \mathrm{~kg} / \mathrm{m}^{3}$. Para a geração de energia é desejável que a biomassa apresente alta densidade, devido à otimização da densidade energética (quantidade de energia por unidade de volume) e diminuição dos custos de transporte. A densidade básica apresentou significativa variação amostral, sendo encontrado alto desvio padrão $\left(59,90 \mathrm{~kg} / \mathrm{m}^{3}\right)$ e alto coeficiente de variação $(34,21 \%)$.

A alta variabilidade dessa propriedade é consequência das diferenças granulométricas e físicas dos resíduos, uma vez que são provenientes de diferentes biomassas e processos industriais e reforça a importância dessa avaliação na seleção de materiais para a produção de diferentes formas de energia. As maravalhas de cedro australiano apresentaram a menor densidade básica $\left(78,39 \mathrm{~kg} / \mathrm{m}^{3}\right)$, já os resíduos do processamento dos grãos de café apresentaram o maior valor para essa propriedade $\left(248,90 \mathrm{~kg} / \mathrm{m}^{3}\right)$.

Quanto à avaliação energética das biomassas, observaram-se comportamentos similares, tanto

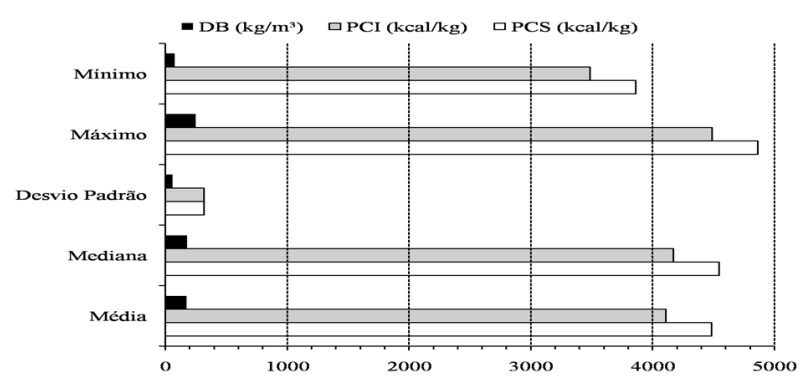

FIGURA 4: Estatísticas descritivas aplicadas às variáveis energéticas e a densidade básica dos resíduos lignocelulósicos.

FIGURE 4: Descriptive statistics applied to energetic variables and basic density of lignocellulosic residues. para o poder calorífico superior (PCS), quanto para o poder calorífico inferior (PCI), uma vez que os resíduos lignocelulósicos avaliados apresentaram teor de hidrogênio (Figura 1) com baixa variação e umidade em base seca de $11 \%$ em média. A polpa celulósica de bambu apresentou os menores valores de PCS e PCI: 4192,40 e 3817,08 kcal/ $\mathrm{kg}$, respectivamente. Já as maravalhas de pinus apresentaram os maiores valores de PCS e PCI: 4864,20 e $4488,88 \mathrm{kcal} / \mathrm{kg}$, respectivamente.

O desvio padrão para o poder calorífico superior (PCS), bem como para o poder calorífico inferior (PCI) foi de $319,11 \mathrm{kcal} / \mathrm{kg}$, resultando em coeficientes de variação inferiores a $8 \%$. No entanto, Brand (2010) afirma que para efeito prático de uso da biomassa para geração de energia são consideradas significativas diferenças superiores a $300 \mathrm{kcal} / \mathrm{kg}$ no poder calorífico dos materiais combustíveis. As diferentes biomassas analisadas apresentaram diferenças superiores à considerada por Brand (2010) no PCS e PCI, sendo que isso deve ser levado em consideração nos cálculos dos sistemas de combustão e geração de energia.

\section{Análise de agrupamento}

Na Figura 5 encontra-se o dendrograma obtido pela análise de agrupamento realizada. Observase que é possível formar quatro grupos distintos se o ponto de corte for considerado na escala das distâncias do dendrograma de 19,42 , que equivale a $67 \%$ da distância total. O primeiro grupo foi formado pelos resíduos da colheita do milho, bagaço de cana-de-açúcar, resíduos do processamento dos grãos de café e maravalhas de eucalipto, enquanto que o segundo grupo foi formado pelas maravalhas de cedro australiano e pinus, o terceiro grupo pela polpa celulósica de bambu e o quarto pela casca de arroz.

Encontrou-se um elevado valor para o coeficiente de correlação cofenética $(0,91)$, ou seja, obteve-se um bom ajuste entre a representação gráfica e a matriz original das distâncias, possibilitando a realização de inferências de interesse com base na avaliação visual da Figura 5.

O grupo composto pelas maravalhas de eucalipto, resíduos do processamento dos grãos de café, resíduos da colheita do milho e pelo bagaço de cana-de-açúcar (Grupo 1) apresentaram-se como intermediários para a produção de energia (Tabela 1). Nota-se que os resíduos que integram esse grupo apresentaram elevado teor de lignina, extrativos totais, C e H. No entanto, o que limita a utilização des- 


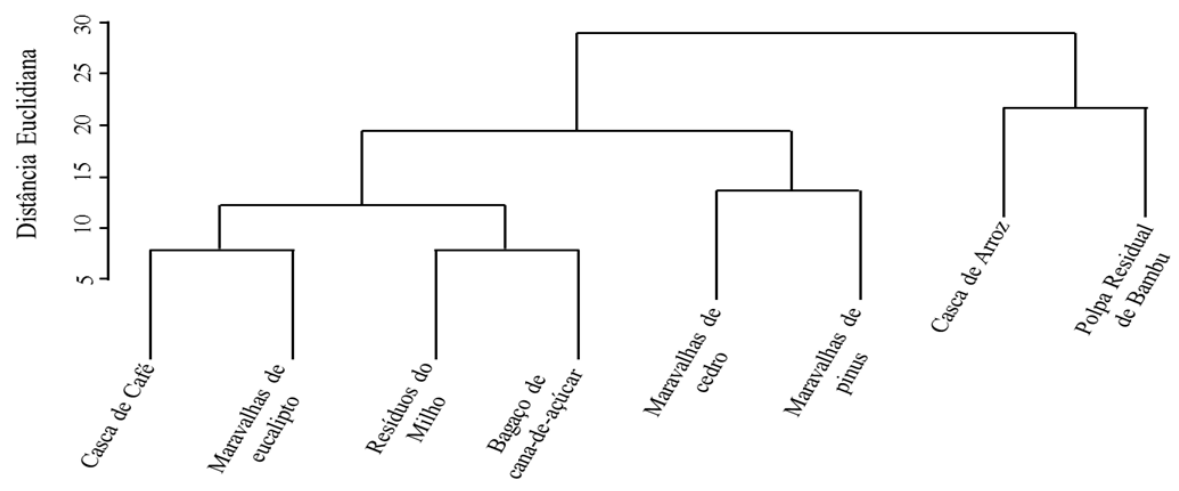

FIGURA 5: Dendrograma da análise de agrupamento.

FIGURE 5: Dendogram of cluster analysis.

TABELA 1: Valores médios das características observadas para os grupos formados pela análise de agrupamento.

TABLE 1: Mean values of the characteristics observed for groups made by cluster analysis.

\begin{tabular}{ccccc}
\hline Características & Grupo 1 & Grupo 2 & Grupo 3 & Grupo 4 \\
\hline Carbono (\%) & 46,68 & 49,50 & 45,20 & 35,74 \\
Hidrogênio (\%) & 6,38 & 6,46 & 6,43 & 5,11 \\
Oxigênio (\%) & 42,41 & 43,05 & 46,67 & 41,56 \\
Nitrogênio (\%) & 1,13 & 0,18 & 0,09 & 0,61 \\
Enxofre (\%) & 0,17 & 0,12 & 0,05 & 0,19 \\
Poder calorífico superior (kcal//kg) & 4551,45 & 4810,20 & 4192,40 & 3863,80 \\
Poder calorífico inferior (kcal/kg) & 4176,13 & 4434,88 & 3817,08 & 3488,48 \\
Densidade básica (kg/m $\left./ \mathrm{m}^{3}\right)$ & 183,70 & 129,83 & 173,67 & 232,52 \\
Lignina (\%) & 26,56 & 27,33 & 8,42 & 28,94 \\
Cinzas (\%) & 3,23 & 0,71 & 1,56 & 16,78 \\
Extrativos totais (\%) & 11,44 & 7,78 & 3,00 & 4,08 \\
\hline
\end{tabular}

ses resíduos são os altos teores de $\mathrm{N}$ (aproximadamente $2 \%$ ) e $\mathrm{S}$ (cerca de $0,23 \%$ ) quantificados para a casca e pergaminho dos grãos de café e resíduos da colheita do milho. A elevada densidade básica encontrada para o Grupo 1 resulta em elevado poder calorífico superior volumétrico $\left(0,84 \mathrm{Gcal} / \mathrm{m}^{3}\right)$, ou seja, maior quantidade de energia por unidade de volume do material combustível.

Já o grupo formado pelas maravalhas de cedro australiano e pinus (Grupo 2) se destaca como potencial para a produção de bioenergia, pois esses resíduos apresentaram, em média, os maiores valores de poder calorífico superior e inferior, altos teores de $\mathrm{C}$ e $\mathrm{H}$, baixos teores de cinzas e $\mathrm{O}$, quando comparados aos demais grupos (Tabela 1). Além disso, as maravalhas de cedro australiano apresentaram alto teor de extrativos totais $(12,94 \%)$. A baixa densidade básica encontrada para o Grupo
2 resulta em baixo poder calorífico superior volumétrico $\left(0,62 \mathrm{Gcal} / \mathrm{m}^{3}\right)$, o que pode ser desfavorável ao aproveitamento energético da biomassa residual que integra esse grupo.

O Grupo 3 formado pela polpa celulósica residual de bambu não apresentou características favoráveis à produção de bioenergia, devido aos baixos teores de extrativos totais e lignina e alto teor de $\mathrm{O}$ e, consequentemente, baixos valores de poder calorífico superior e inferior (Tabela 1). No entanto, o poder calorífico superior volumétrico desse grupo $\left(0,73 \mathrm{Gcal} / \mathrm{m}^{3}\right)$ foi maior que o encontrado para o Grupo 2.

O grupo formado pela casca de arroz (Grupo 4) também não apresentou características favoráveis à produção de energia devido aos baixos teores de $\mathrm{C}$ e $\mathrm{H}$, aos elevados teores de cinzas, $\mathrm{N}$ e $\mathrm{S}$, o que resultou em baixos valores de poder calorífico 
superior e inferior (Tabela 1). No entanto, a casca de arroz apresentou o maior poder calorífico superior volumétrico $\left(0,90 \mathrm{Gcal} / \mathrm{m}^{3}\right)$, mas o seu elevado teor de cinzas pode causar problemas de corrosão nos equipamentos metálicos. Além disso, as cinzas não se queimam permanecendo no local do processo e exigindo um sistema adequado para a sua retirada (BRAND, 2010).

\section{Análise de componentes principais}

Na Figura 6 encontram-se a variância explicada acumulada e os autovalores (scree plot) das componentes principais obtidas por meio da matriz de correlação das variáveis originais (teores de cinzas, carbono, hidrogênio, enxofre, oxigênio, poder calorífico superior e poder calorífico inferior).

Observa-se que as duas primeiras componentes explicam aproximadamente $93 \%$ da variância. Analisando-se os autovalores observa-se que é possível considerar apenas 2 ou 3 componentes principais, pois a partir desse valor não há grandes alterações nos valores de suas variâncias. Entretanto, optou-se por considerar apenas duas componentes principais devido à grande parcela da variância total dos dados explicada por elas, ou seja, as informações mais relevantes dos dados amostrais originais estão contidas nas duas primeiras componentes principais.

Os autovetores normalizados e as correlações entre as variáveis originais e as componentes principais 1 e 2 encontram-se apresentados na Tabela 2.

A primeira componente principal é basicamente um índice de desempenho global para a ava-

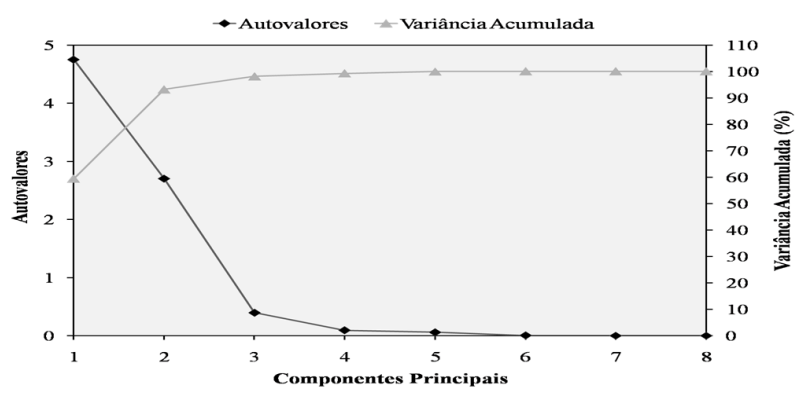

FIGURA 6: Variância explicada acumulada e autovalores obtidos da matriz de correlação.

FIGURE 6: Variance explained cumulative and eigenvalues obtained by correlation matrix. liação da viabilidade do aproveitamento energético da biomassa (ou uma forma de contraste). Os mais elevados coeficientes da componente principal, em módulo, são relativos ao PCS, PCI, C, H e cinzas, sendo dominada por essa última variável. Observase que quanto maior os teores de cinzas da biomassa vegetal maior será o valor numérico da componente (escore), sendo o contrário determinado para os teores de $\mathrm{C}$ e H e para o PCS e PCI. Logo, percebe-se que essa componente reúne as principais características necessárias para a seleção e avaliação preliminar de materiais lignocelulósicos para a produção de bioenergia. Observa-se que as maiores correlações, em módulo, foram observadas para os teores de cinzas, carbono e hidrogênio e para o poder calorífico superior e inferior.

A segunda componente principal pode ser interpretada como sendo um contraste entre os teores de $\mathrm{N}$ e $\mathrm{S}$ e teor de $\mathrm{O}$, ou seja, é basicamente um índice que pode refletir em poluição ambiental e não aproveitamento energético da biomassa vegetal, uma vez que altos valores dessa componente (escore) estão associados: a) a altos teores de $\mathrm{O}$, característica esta não favorável para a produção de bioenergia (HUANG et al., 2009; PAULA et al., 2011; PROTÁSIO et al., 2011; NORDIN, 1994) e; b) baixos escores estão associados a altos teores de $\mathrm{N}$ e $\mathrm{S}$ na biomassa que podem resultar em poluição ambiental (KUMAR et al., 2010; BILGEN e KAYGUSUZ, 2008). Percebe-se então que essa componente principal encontra-se altamente correlacionada, em módulo, com os teores de $\mathrm{O}, \mathrm{S}$ e $\mathrm{N}$.

$\mathrm{Na}$ Figura 7 encontram-se o diagrama de ordenação das variáveis originais e os escores das duas primeiras componentes principais. Um ponto qualquer plotado no diagrama (representando um resíduo lignocelulósico) pode ser relacionado com cada seta (representando as variáveis originais observadas), por meio de uma perpendicular partindo da linha da seta até o referido ponto (ALVARENGA e DAVIDE, 1999).

Pelos valores dos escores calculados para a primeira componente principal, percebe-se que os resíduos florestais se destacaram em relação aos agrícolas quanto à viabilidade do aproveitamento energético. As maravalhas de pinus, cedro australiano e eucalipto apresentaram os menores escores, respectivamente, dado aos baixos teores de cinzas quantificados para esses materiais. As maravalhas de pinus e cedro apresentaram ainda altos valores de PCS e PCI e altos teores de $\mathrm{C}$ e $\mathrm{H}$, quando comparados à média geral. A polpa celulósica residual de 
TABELA 2: Autovetores normalizados e as correlações entre as variáveis originais e as componentes principais.

TABLE 2: Normalized eigenvectors and correlations between original variables and principle components.

\begin{tabular}{ccccc}
\hline \multirow{2}{*}{ Variáveis originais } & \multicolumn{2}{c}{ Componente Principal 1 } & \multicolumn{2}{c}{ Componente Principal 2 } \\
\cline { 2 - 5 } & $\hat{e}_{1}$ & $\mathrm{r}$ & $\hat{e}_{2}$ & $\mathrm{r}$ \\
\hline Cinzas & 0,449 & 0,97 & $-0,055$ & $-0,09$ \\
Poder calorífico superior & $-0,393$ & $-0,86$ & $-0,276$ & $-0,45$ \\
Poder calorífico inferior & $-0,393$ & $-0,86$ & $-0,276$ & $-0,45$ \\
Carbono & $-0,442$ & $-0,96$ & $-0,127$ & $-0,21$ \\
Nitrogênio & 0,158 & 0,34 & $-0,529$ & $-0,87$ \\
Hidrogênio & $-0,409$ & $-0,89$ & $-0,151$ & $-0,25$ \\
Enxofre & 0,252 & 0,55 & $-0,495$ & $-0,81$ \\
Oxigênio & $-0,193$ & $-0,42$ & 0,529 & 0,87 \\
\hline
\end{tabular}

Em que: $\hat{e}_{1,} \hat{e}_{2:}$ autovetores das componentes principais $1 \mathrm{e} 2$, respectivamente; : correlação entre as variáveis originais $\mathrm{e}$ as componentes principais.

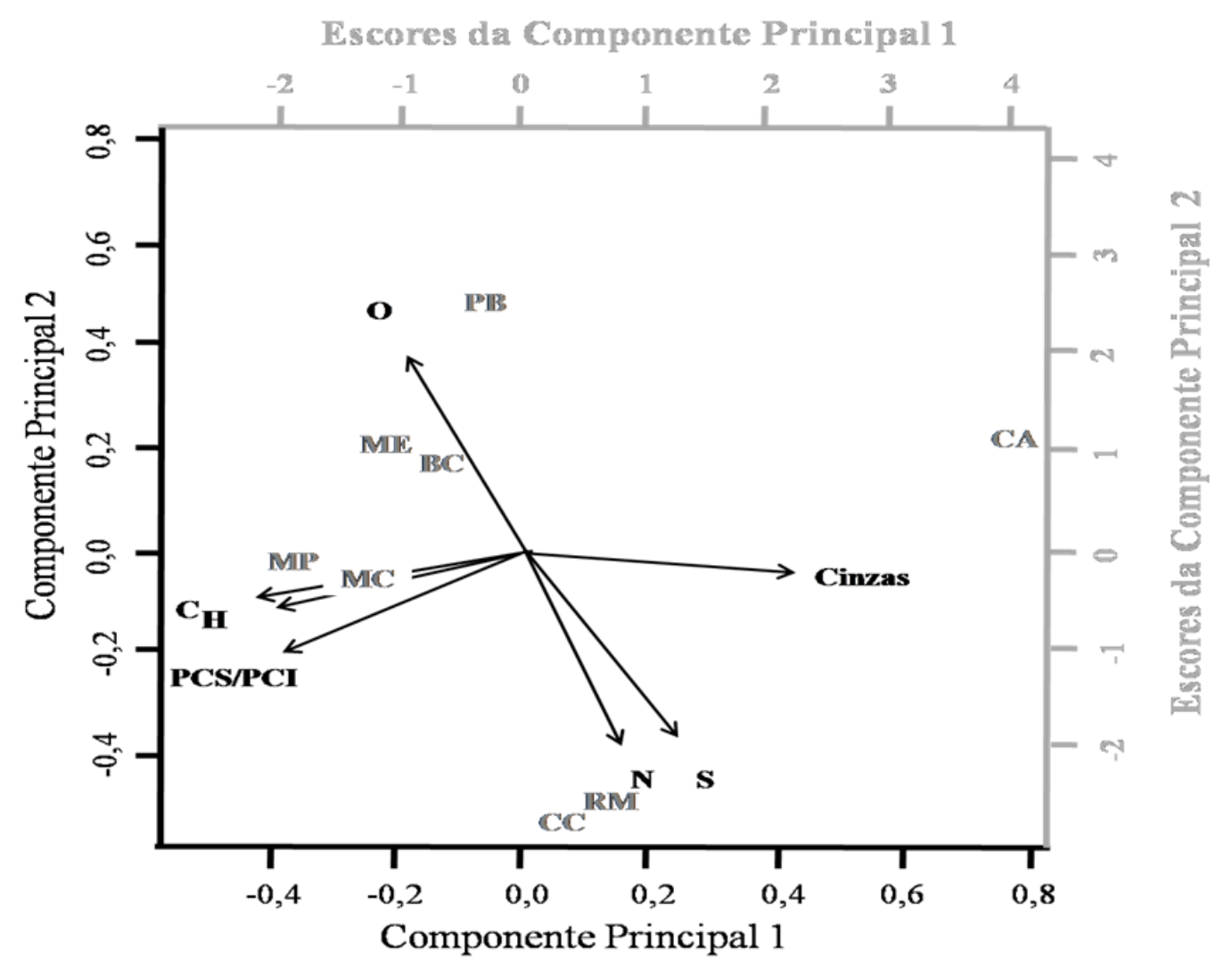

Em que: PCS: poder calorífico superior; PCI: poder calorífico inferior; C: carbono; H: hidrogênio; O: oxigênio; N: nitrogênio; S: enxofre; MP: maravalhas de pinus; MC: maravalhas de cedro; $\mathrm{CC}$ : casca e pergaminho dos grãos de café; RM: resíduos do milho; ME: maravalhas de eucalipto; BC: bagaço de cana-de-açúcar; PB: polpa celulósica residual de bambu; CA: casca de arroz.

FIGURA 7: Diagrama de ordenação das variáveis originais e escores das duas primeiras componentes principais.

FIGURE 7: Diagram of original variables ordination and scores of the two first principle components. 
bambu e o bagaço de cana-de-açúcar apresentaram posição intermediária. A casca de arroz, resíduos do milho e a casca de café apresentaram os mais elevados escores para a componente principal 1 , em virtude dos elevados teores de cinzas desses materiais.

Os menores escores da segunda componente principal foram observados para os resíduos do processamento do café e da colheita do milho, respectivamente, devido aos elevados teores de $\mathrm{N}$ e $\mathrm{S}$.

A polpa celulósica de bambu, a casca de arroz, as maravalhas de eucalipto e o bagaço de cana-de-açúcar apresentaram os maiores escores da componente principal 2, devido aos elevados teores de $\mathrm{O}$ e baixos teores de $\mathrm{N}$ e $\mathrm{S}$ quando comparados aos demais resíduos lignocelulósicos. As maravalhas de pinus e cedro apresentaram valores próximos de zero, pois apresentaram baixos valores de $\mathrm{O}$, $\mathrm{N}$ e $\mathrm{S}$ quando comparados aos demais.

Observa-se que resultados similares podem ser obtidos se forem considerados os grupos formados pela análise de agrupamento e os grupos que podem ser formados pelos escores das duas componentes principais consideradas, ou seja, nota-se que a casca de arroz forma um grupo distinto. Já as maravalhas de cedro e pinus podem ser agrupadas em um único grupo, pois apresentaram características similares. Os resíduos da colheita do milho e a casca de café apresentaram-se heterogêneos em relação às maravalhas de eucalipto e ao bagaço de cana-de-açúcar com base nos escores das duas primeiras componentes principais, diferindo do encontrado na análise de agrupamento. Com isso, pode-se formar um grupo entre as maravalhas de eucalipto, bagaço de cana-de-açúcar e polpa celulósica residual de bambu. Recomenda-se a utilização de outra medida de proximidade para a análise de agrupamento como a distância de Mahalanobis.

A análise de agrupamento e a análise de componentes principais foram eficientes na avaliação e seleção de resíduos lignocelulósicos para a produção de bioenergia, mas o agrupamento obtido pela análise de componentes principais foi mais eficiente. Além disso, para a análise de componentes principais foi necessário um menor número de variáveis, o que permitiu o agrupamento dos resíduos em grupos mais homogêneos para a produção de energia.

\section{CONCLUSÕES}

As duas técnicas multivariadas empregadas para a avaliação e seleção de resíduos lignocelu- lósicos para a produção de energia foram eficientes, sendo observadas semelhanças entre os grupos de biomassa formados pelos escores da análise de componentes principais e pelo dendrograma obtido pela análise de agrupamento. Pela interpretação da primeira componente principal obtida, criou-se um índice de desempenho global para avaliar a viabilidade do aproveitamento energético da biomassa. Já a interpretação da segunda componente principal permitiu um contraste entre os teores de nitrogênio e enxofre e o teor de oxigênio.

\section{REFERÊNCIAS BIBLIOGRÁFICAS}

AKKAYA, A. V. Proximate analysis based multiple regression models for higher heating value estimation of low rank coals. Fuel Processing Technology, v. 90, n. 2, p. 165-170, 2009.

ALVARENGA, M. I. N.; DAVIDE, A. C. Características físicas e químicas de um Latossolo Vermelho-Escuro e a sustentabilidade de agroecossistemas. Revista Brasileira de Ciência do Solo, v. 23, p. 933-942, 1999.

ASSOCIAÇÃO BRASILEIRA DE NORMAS TÉCNICAS. NBR 8633: Carvão Vegetal determinação do poder calorífico. Rio de Janeiro, Brasil, 1984.

ASSOCIAÇÃO BRASILEIRA TÉCNICA DE CELULOSE E PAPEL. M11/77: teor de cinzas. São Paulo, 1974a. 8 p.

ASSOCIAÇÃO BRASILEIRA TÉCNICA DE CELULOSE E PAPEL. M3/69: Métodos de ensaio. São Paulo, Brasil, 1974b. 8p.

ASSOCIAÇÃO BRASILEIRA TÉCNICA DE CELULOSE E PAPEL. M70/71: Métodos de ensaio. São Paulo, Brasil, 1974c. 8p.

AZZINI, A.; SALGADO, A. L. B.; TEIXEIRA, J. P.

F. Curva de maturação da Crotalarea juncea L. em função da densidade do caule. Bragantia, v. 40, n. 1, p. 1-10, 1981.

BECH, N.; JENSEN, P. A.; DAM-JOHANSEN, $\mathrm{K}$. Determining the elemental composition of fuels by bomb calorimetry and the inverse correlation of HHV with elemental composition. Biomass and Bioenergy, v. 33, n. 3, p. $534-537,2009$.

BILGEN, S.; KAYGUSUZ, K. The calculation of the chemical exergies of coal-based fuels by using the higher heating values. Applied Energy, v. 85, n. 8, p. $776-785,2008$.

BRAND, M. A. Energia de biomassa florestal. Rio de Janeiro: Interciência, 2010. 131 p.

CAIXETA, R. P. et al. Propriedades e classificação 
da madeira aplicadas à seleção de genótipos de Eucalyptus. Revista Árvore, v. 27, n. 1, p. 43-51, 2003.

CORDERO, T. et al. Predicting heating values of lignocellulosics and carbonaceous materials from proximate analysis. Fuel, v. 80 , n. 1, p. 1567$157,2001$.

DEMIRBAS, A. Relationships between lignin contents and heating values of biomass. Energy Conversion and Management, v. 42, n. 2, p. 183188, 2001.

DEMIRBAS, A; DEMIRBAS, H. A. Estimating the calorific values of lignocellulosic fuels. Journal Energy, Exploration \& Exploitation, v. 20, n. 1, p. 105-111, 2004.

EROL, M.; HAYKIRI-ACMA H.; KUÇUKBAYRAK, S. Calorific value estimation of biomass from their proximate analyses data. Renewable Energy, Oxford, v. 35, n. 1, p. 170 173, 2010.

FERREIRA, D. F. Estatística Multivariada. 1. ed. Lavras: UFLA, 2008. 662 p.

FRIEDL, A. et al. Prediction of heating values of biomass fuel from elemental composition. Analytica Chimica Acta, v. 544, n. 1-2, p. 191-198, 2005.

GANI, A.; NARUSE, I. Effect of cellulose and lignin content on pyrolysis and combustion characteristics for several types of biomass. Renewable Energy, Oxford, v. 32, n. 4, p. 649-661, 2007.

GÓMEZ, C. J. et al. Thermogravimetry/mass spectrometry study of woody residues and an herbaceous biomass crop using PCA techniques. Journal Anaytical Applied Pyrolysis, v. 80, n. 2, p. 416-426, 2007.

HAIR JR., J. F. et al. Análise multivariada de dados. 6. ed. Porto Alegre: Bookman, 2009. 688 p.

HUANG, C. et al. Ultimate analysis and heating value prediction of straw by near infrared spectroscopy. Waste Management, v. 29, n. 6, p.1793-1797, 2009.

JOHNSON, R. A; WICHERN, D. W. Applied multivariate statistical analysis. New Jersey: Prentice-Hall, 1992. 607 p.

KENT, M.; COKER, P. Vegetation description and analysis. Baffins Lane, John Wiley \& Sons, 1992. $363 \mathrm{p}$.

KUMAR, R. et al.. Effect of tree-age on calorific value and other fuel properties of Eucalyptus hybrid. Journal of Forestry Research, v. 21, n. 4, p.514 516, 2010.

KUMAR, M.; GUPTA, R. C.; SHARMA, T. Effect of carbonisation conditions on the yield and chemical composition of Acacia and Eucalyptus wood chars. Biomass and Bioenergy, v. 3, n. 3, p. 411-417, 1992.

MAJUMDER, A. K. et al. Development of a new proximate analysis based correlation to predict calorific value of coal. Fuel, v. 87, n. 13-14, p. 3077 3081, 2008.

MARRIOTT, F. H. C. The interpretation of multiple observation. New York: Academic Press, 1974. $117 \mathrm{p}$.

MINGOTI, S. A. Análise de dados através de métodos de estatística multivariada: uma abordagem aplicada. Belo Horizonte: UFMG, 2005. $297 \mathrm{p}$.

MINISTÉRIO DE MINAS E ENERGIA. Disponível em: http://www.mme.gov.br/mme/menu/todas publicacoes.html . Acesso em 15 de Setembro de 2010.

NORDIN, A. Chemical and elemental characteristics of biomass fuels. Biomass and Bioenergy, v. 6, n. 5, p. 339-347, 1994.

PARIKH, J.; CHANNIWALA, S. A.; GHOSAL, G. $\mathrm{K}$. A correlation for calculating $\mathrm{HHV}$ from proximate analysis of solid fuels. Fuel, v. 84, n. 5, p. 487-494, 2005.

PAULA, L. E. R. et al. Characterization of residues from plant biomass for use in energy generation. Cerne, Lavras, v. 17, n. 2, p. 237-246, 2011.

PIMENTEL-GOMES, F. Curso de estatística experimental. 15. ed. Piracicaba: FEALQ, 2009. $451 \mathrm{p}$.

PROTÁSIO, T. P. et al. Relação entre o poder calorífico superior e os componentes elementares e minerais da biomassa vegetal. Pesquisa Florestal Brasileira, Colombo, v. 31, n. 66, p. 122 -133, 2011. DOI: 10.4336/2011.pfb.31.66.113

R DEVELOPMENT CORE TEAM. R: A language and environment for statistical computing. $R$ Foundation for Statistical Computing, Vienna, Austria. Disponível em: $<$ http://www.R-project. org $>$. Acesso em 10 de Dezembro de 2010.

SHAFIZADEH, F. Basic principles of direct combustion. In: Sofer SS, Zabrosky OR (ed.). Biomass conversion process for energy and fuels. New York: Plenum Press, 1981. p. 103-112.

SHENG, C.; AZEVEDO , J. L. T. Estimating the higher heating value of biomass fuels from basic analysis data. Biomass and Bioenergy, v. 28, n. 5, p. 499-507, 2005

TELMO, C.; LOUSADA, J. Heating values of wood pellets from different species. Biomass and Bioenergy, v. 35, n. 7, p. 2634-2639, 2011. 